\title{
Écologie des STOMOXEs (DIPTERA : MUSCIDAE) aU GABON. I - Premier InVENTAIRe dans difFérentes ZONeS ÉCOLOgIQUeS
}

\author{
MAVOUNGOU J.F.****, JAY-ROBERT P.**, GILLES J.***, ATSAME EDDA A.* \& DUVALLET G.**
}

Summary: ECOlogy of STOMOXYS Flies (Diptera: Muscidae) in GABON. I. First SURVEY IN DIFFERENT ECOlOGICAL AREAS

The stomoxyine flies are hematophagous diptera and potential vectors of various pathogenic agents. Like those of the Afrotropical Region, the stomoxyine flies of Gabon remain nearly unknown. For these reasons, an entomological survey was conducted in a transverse way in eight localities representative of the various ecological zones of Gabon. The survey was based on the use of Vavoua traps. Various environmental factors able to influence the captures were noticed and included into a canonical correspondence analysis. In total, 15,966 Stomoxys spp., belonging to seven species or sub-species, were captured. The apparent densities (DAP) expressed as the number of flies per trap and per day, were highest in Franceville (41), Bakoumba (40), Makokou (25) and Mouila (21). The most abundant species were S. n niger (33.4\%), S. transvittatus (33\%), then S. calcitrans $(17 \%)$. The principal factors that could explain the variability of the captures were the degree of anthropisation, the botanical facies (savanna or forest), the presence of wild and domestic fauna and the nature of the vegetal cover of the ground. S. calcitrans, S. niger niger were abundant in the areas where human presence was manifest. S. xanthomelas was present in forest belts. S. transvittatus, S. omega, S. inornatus were ubiquitous species. S. niger bilineatus was found in savannas areas.

KEY WORDS : Stomoxys spp., ecology, distribution, Gabon

\section{INTRODUCTION}

L es stomoxes, regroupés dans le genre Stomoxys Geoffroy, 1762, sont des diptères Muscidae, appartenant à la sous-famille des Stomoxyinae. Cette sous-famille comprend au total 10 genres différents (Zumpt, 1973). Ce sont des mouches hématophages, de 3 à $10 \mathrm{~mm}$ de longueur, ayant l'aspect général d'une mouche domestique, mais dotées d'un proboscis piqueur-

\footnotetext{
* Institut de Recherche en Écologie Tropicale (IRET), BP 13354, Libreville, Gabon.

** UMR 5175 CEFE (Centre d'Écologie Fonctionnelle et Évolutive), Université de Montpellier Languedoc-Roussillon, Montpellier, France. **** Institut für Vergleichende Tropenmedizin und Parasitologie, München, Germany

Correspondance: Jacques F. Mavoungou, UMR 5175 (CEFE), Université Paul-Valéry, 34199 Montpellier Cedex 5, France.

E-Mail : mavoungoujacques@yahoo.fr
}

\begin{abstract}
Résumé :
Les stomoxes (Stomoxys spp.) sont des diptères hématophages, vecteurs potentiels de divers agents pathogènes. Comme ceux de la région afrotropicale en général, les stomoxes du Gabon demeurent mal connus. Pour ces raisons, une enquête entomologique a été conduite de façon transversale dans huit localités représentatives des diverses zones écologiques du Gabon. L'enquête est basée sur l'utilisation de pièges Vavoua. Divers facteurs environnementaux pouvant influer sur les captures ont été relevés et inclus dans une analyse canonique des correspondances. Au total, 15966 stomoxes, appartenant à sept espèces ou sous-espèces, ont été capturés. Les densités apparentes (DAP), exprimées en nombre de stomoxes par piège et par jour, sont importantes dans les localités de Franceville (4 1), Bakoumba (40), Makokou (25) et Mouila (21). Les espèces les plus abondantes sont Stomoxys n. niger $133,4 \%$ de l'ensemble des captures), S. transvittatus (33\%) puis S. calcitrans (17\%). Les principaux facteurs qui expliquent la variabilité des captures sont le degré d'anthropisation du milieu, le faciès botanique (savane ou forêt), la présence de la faune sauvage et domestique et la nature de la couverture végétale du sol. S. calcitrans et S. n. niger sont abondants dans les zones où la présence humaine est manifeste. S. xanthomelas est inféodé aux zones forestières. S. transvittatus, $\mathrm{S}$. omega, et $\mathrm{S}$. inornatus sont des espèces ubiquistes. S. niger bilineatus se retrouve dans les zones de savane.
\end{abstract}

MOTS CLÉS : Stomoxys spp., écologie, distribution, Gabon.

suceur. Le genre Stomoxys comprend 18 espèces dont 17 sont tropicales (régions afrotropicale et orientale) et une seule est cosmopolite : Stomoxys calcitrans (Linné, 1758). Ces insectes, en raison de leur hématophagie, représentent un fléau à la fois par leur nuisance directe (harcèlement et prédation sanguine), mais aussi par leur rôle de vecteur potentiel de divers agents pathogènes (Leclercq, 1971; Zumpt, 1973; Mihok et al., 1995a; Foil \& Gorham, 2000).

Les stomoxes de la région afrotropicale demeurent mal connus. Malgré l'existence de travaux dans quelques localités (Hafez et al., 1959a et b, 1961; Harley, 1965; Zumpt, 1973; Kangwagye, 1973; Mihok et al., 1996a, 1996b; Charlwood \& Sama, 1996), les connaissances écologiques et biologiques portant sur les différentes espèces de ce groupe demeurent fragmentaires. De plus, la majorité des informations disponibles a été obtenue à partir de l'espèce cosmopolite $S$. calcitrans et 
plus récemment à partir de l'espèce $S$. niger niger Macquart, 1851 (Gilles, 2005).

Par sa position géographique en Afrique équatoriale, le Gabon fait partie des pays à forte biodiversité, dont la préservation a été prise en compte par la création récente de treize parcs nationaux qui occupent $10 \%$ de la superficie du pays. Cependant l'entomofaune est encore peu connue, malgré les travaux de Basset et al. (2004) dans les aires protégées de Gamba. Les recherches sur les stomoxes entreprises au Gabon entrent dans le cadre de l'étude de la diversité de l'entomofaune, notamment dans les aires protégées, mais aussi dans celui de l'étude des insectes potentiellement vecteurs de pathogènes. La connaissance de la distribution et de l'écologie des différentes espèces de stomoxes sont des éléments nécessaires à l'élaboration de stratégies de contrôle de ces insectes en cas de transmission de pathogènes.

Pour recueillir des données sur leur distribution et abondance relative, une enquête entomologique a été réalisée dans plusieurs localités représentatives des différentes zones écologiques du Gabon.

\section{MATÉRIELS ET MÉTHODES}

\section{Milieu D'ÉTUde}

L e Gabon est situé en Afrique centrale (Golfe de Guinée) entre $3^{\circ}$ Nord et $4^{\circ}$ Sud en latitude et entre $\mathcal{U}^{\circ}$ et $14^{\circ}$ Est en longitude. Sa superficie totale est de $267667 \mathrm{~km}^{2}$. Sa population est estimée à 1,2 millions d'habitants. Son climat est de type équatorial, caractérisé par une température moyenne annuelle de $26^{\circ} \mathrm{C}$, une forte humidité et d'importantes précipitations. La pluviométrie moyenne annuelle varie de 1500 à $3000 \mathrm{~mm}$ et l'hygrométrie moyenne est toujours supérieure à $80 \%$. Quatre saisons se succèdent : une petite saison sèche de décembre à janvier, une grande saison des pluies de février à mai, une grande saison sèche de juin à septembre et une petite saison des pluies d'octobre à novembre. La forêt et la savane sont les deux principales formations végétales du Gabon. La forêt s'étend sur près de $85 \%$ de la superficie totale du pays (Edicef, 1983). Les $15 \%$ restant du territoire constituent le domaine non forestier, essentiellement couvert de steppes et de savanes, auxquelles s'ajoutent les espaces occupés par les hommes (villes, villages, campements).

Les prospections de l'enquête présentée ici ont été réalisées dans huit localités (figure 1) : Bakoumba et Franceville dans la province du Haut Ogooué, Mouila et Fougamou dans la province de Ngounié, Makokou et Malouma dans la province d'Ogooué Ivindo, Libreville et Ntoum dans la province de l'Estuaire. Ces localités ont été choisies pour des raisons pratiques et logistiques d'accessibilité dans les principales zones écologiques du Gabon identifiées par les formations végétales dominantes (Richard \& Léonard, 1993). Bakoumba et Franceville représentent des zones de savane, Mouila et Fougamou des zones de mosaïque forêt-savane, Makokou et Malouma des zones de forêt, enfin Libreville et Ntoum des espaces périurbanisés.

\section{CAPTURE Des STOMOXES}

Les stomoxes ont été capturés à l'aide de pièges Vavoua (Laveissière \& Grébaut, 1990). Ce piège, initialement mis au point en Afrique pour capturer des glossines, a montré également son efficacité pour les stomoxes à l'île de la Réunion (Gilles et al., 2007). Toutes les captures ont été réalisées entre février et mai 2005, c'est-à-dire pendant la grande saison des pluies. Suivant l'importance de la zone prospectée, 12 à 16 pièges Vavoua ont été utilisés pour chaque localité (114 pièges au total). Chaque piège était activé le matin avant $7 \mathrm{~h}$ et relevé le soir après $19 \mathrm{~h}$ pendant sept jours consécutifs. Les cages ont été étiquetées avec le numéro du piège et ramenées au laboratoire. Elles ont été ensuite placées dans un congélateur durant 15 minutes pour tuer les mouches avant de les stocker dans des flacons contenant de l'éthanol à $95^{\circ}$.

\section{ENVIRONNEMENT DES PIÈGES}

Les pièges ont été disposés de manière à prendre en compte la diversité des habitats de chaque localité. Les paramètres de l'environnement de chacun des pièges, susceptibles d'influencer l'abondance des stomoxes, ont été relevés. Pour cela, quatre descripteurs environnementaux ont été considérés : la densité humaine, la présence de faune vertébrée, le type de paysage et la couverture du sol. Les descripteurs et leurs modalités sont décrits dans le tableau I. Ces descripteurs représentent

\begin{tabular}{|c|c|c|c|}
\hline \multicolumn{3}{|c|}{ Échelle du paysage (rayon de $500 \mathrm{~m}$ ) } & \multirow{2}{*}{$\begin{array}{c}\text { Environnement immédiat } \\
\text { Couverture du sol }\end{array}$} \\
\hline Densité de population & Faune vertébrée & Paysage & \\
\hline Zone inhabitée (pop/0) & Aucune $(\mathrm{fau} / 0)$ & Forêt (for) & Matière organique (sol/MO) \\
\hline $1-10$ pers. $(\mathrm{pop} / 1-10)$ & Faune sauvage (fau/s) & Savane (sav) & Tapis herbacé $(\mathrm{sol} / \mathrm{H})$ \\
\hline $11-20$ pers. (pop/11-20) & Faune domestique (fau/d) & Cabane (cab) & Champs de culture (sol/C) \\
\hline $21-50$ pers. (pop/21-50) & Les deux $(\mathrm{fau} / \mathrm{ds})$ & Maison (mai) & Pâturage $(\mathrm{sol} / \mathrm{P})$ \\
\hline
\end{tabular}

Tableau I. - Paramètres utilisés pour caractériser l'environnement des pièges (entre parenthèses sont indiquées les abréviations utilisées pour l'ACC). 


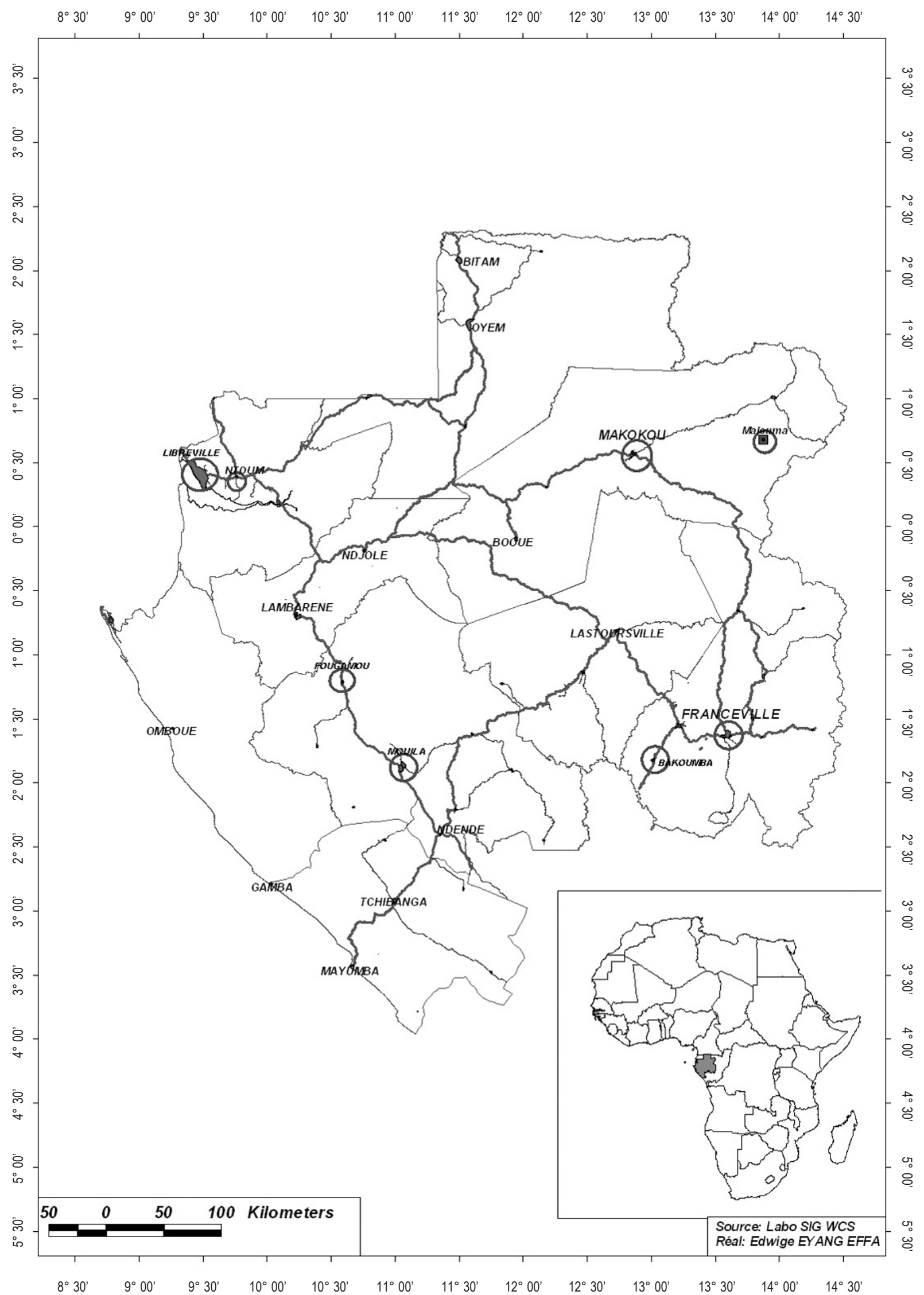

Fig. 1. - Carte du Gabon. Les cercles indiquent les localités prospectées. 
deux échelles différentes de perception de l'environnement des pièges : la densité humaine, la présence de faune vertébrée et le paysage correspondent à une échelle moyenne ( $500 \mathrm{~m}$ autour du piège), la couverture du sol concerne l'environnement immédiat des pièges (quelques mètres).

\section{IDENTIFICATION DES STOMOXES}

Les insectes ont été triés au fur et à mesure de leur arrivée au laboratoire. Une première séparation était faite entre stomoxes et autres diptères (glossines, mouches domestiques, tabanides). La discrimination entre les différentes espèces de stomoxes a été faite ensuite à l'aide de la clé de Zumpt (1973), et du caractère morphologique complémentaire décrit par Garros et al. (2004) pour mieux séparer $S$. calcitrans et $S$. niger. Après tri, identification et dénombrement, les différentes espèces de stomoxes et les autres diptères ont été conservés dans de l'éthanol à $95^{\circ}$ pour des études moléculaires ultérieures.

\section{ANALYSE DES DONNÉES}

L'abondance des stomoxes est traduite par la densité apparente par piège (DAP), exprimée en nombre de stomoxes capturés par piège et par jour.

Une analyse canonique des correspondances (CANOCO 4.5; ter Braak, 1986; ter Braak \& Smilauer, 2002) a été réalisée afin d'identifier les paramètres influençant la distribution des espèces. La matrice faunistique utilisée rassemble les observations des sept espèces de stomoxes $(\log$ effectif +1$)$ dans les 112 pièges ayant collecté des insectes (deux pièges n'ont capturé aucun stomoxe). La matrice des descripteurs (codée en présence-absence) caractérise chaque piège d'après 1) sa localité (huit prospectées) et 2) les paramètres environnementaux décrits dans le tableau I.

\section{RÉSULTATS}

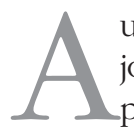

u total 114 pièges Vavoua ont été placés sept jours dans les huit localités prospectées, soit 798 pièges-jours. Sur un total général de 21830 insectes collectés, on a dénombré 20428 diptères dont 15966 stomoxes. Les autres diptères capturés étaient essentiellement : 1) des Haematobosca spp. (Muscidae : Stomoxyinae) et des glossines (Glossinidae) dans la localité de Bakoumba, 2) des tabanides (Tabanidae) capturés principalement à Makokou et à Malouma. La répartition des captures et les densités apparentes des stomoxes par piège (DAP) entre les différentes localités sont présentées dans la figure 2. Les captures de stomoxes ont été plus importantes à Franceville et Bakoumba (environ 40 stomoxes/piège/jour). À Makokou et à Mouila les DAP étaient de 24 et 20 respecti- vement; à Libreville et Ntoum elles étaient de 11 et 10 respectivement. Enfin, à Fougamou et Malouma, peu d'insectes ont pu être observés (DAP de 7 et 5 respectivement).

L'identification des stomoxes a permis de mettre en évidence la présence de sept espèces et sous-espèces. Le pourcentage de chaque espèce dans l'ensemble des captures est présenté dans la figure 3. Les espèces les plus abondantes sont Stomoxys niger niger Macquart, 1851 (33,4\%) et S. transvittatus Villeneuve, 1916 (33\%). Les espèces S. calcitrans (16,7\%), S. inornatus Grünberg, 1906 (6,7\%), S. n. bilineatus Grünberg, 1906 (5\%) et $S$. omega Newstead, 1907 (4,8\%) apparaissent comme moins abondantes. S. xanthomelas Roubaud, 1937 fut très rarement observé.

La distribution et l'abondance des différentes espèces dans les 8 localités sont indiquées dans le tableau II.

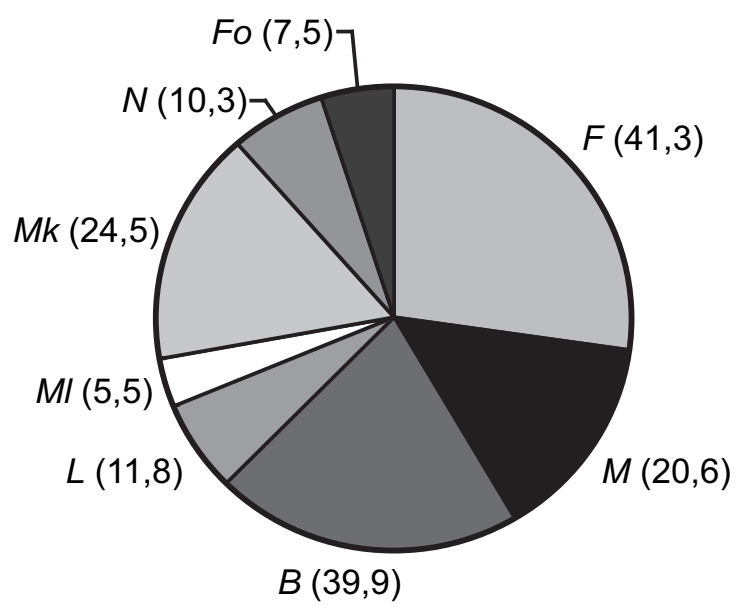

Fig. 2. - Répartition des captures de Stomoxys spp. entre les différentes localités ( $F$ : Franceville, $M$ : Mouila, $B$ : Bakoumba, $L$ : Libreville, $M l$ : Malouma, $M k$ : Makokou, $N$ : Ntoum, $F o$ : Fougamou). Pour chaque localité est indiqué entre parenthèses le nombre de stomoxes capturés par piège et par jour (densité apparente, DAP).

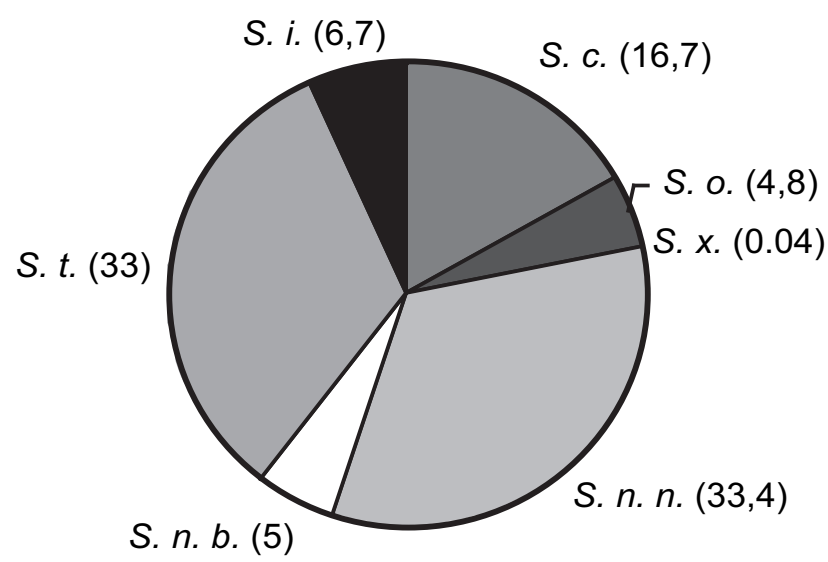

Fig. 3. - Répartition des captures des différentes espèces de Stomoxys (S. t. : Stomoxys transvittatus, S. c. : S. calcitrans, S. n. n. : S. niger niger, S. n. b. : S. niger bilineatus, S. o.: S. omega, S. i. : S. inornatus, $S . x$. : S. xanthomelas), Pour chaque espèce, le pourcentage sur l'ensemble des captures est indiqué entre parenthèses. 


\section{Espèces}

$\begin{array}{ccccccc}\text { S. calcitrans } & \text { S.n. niger } & \text { S.n. bilineatus } & \text { S. transvittatus } & \text { S. inornatus } & \text { S. omega } & \text { S. xanthomelas } \\ \text { Linné } 1758 & \text { Macquart } 1851 & \text { Grünberg } 1906 & \text { Villeneuve } 1916 & \text { Grünberg } 1906 & \text { Newstead } 1907 & \text { Roubaud } 1937\end{array}$

Localités

Bakoumba

Fougamou

Franceville

Libreville

Makokou

Malouma

Mouila

Ntoum

$\begin{array}{cc}+ & +++ \\ ++ & +++ \\ +++ & ++++ \\ +++ & +++ \\ + & + \\ + & + \\ + & +++ \\ +++ & +++\end{array}$

++
+
+
+
+
+

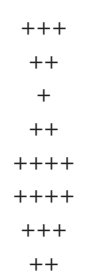

$++$

$\begin{array}{cc}++ & + \\ ++ & + \\ + & + \\ ++ & + \\ +++ & + \\ +++ & + \\ ++ & + \\ ++ & +\end{array}$

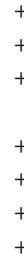

Rien : $0 \%,+<12,5 \%,++12,5-25 \%,+++25-50 \%,++++>50 \%$ des stomoxes capturés dans la localité.

Tableau II. - Distribution et abondance relative des espèces dans les différentes localités.

\begin{tabular}{|c|c|c|c|c|c|c|c|c|c|c|c|c|c|c|c|c|}
\hline & \multicolumn{4}{|c|}{ Population } & \multicolumn{4}{|c|}{ Faune } & \multicolumn{4}{|c|}{ Paysage } & \multicolumn{4}{|c|}{ Sol } \\
\hline & Pop/0 & $\begin{array}{c}\text { Pop/ } \\
1-10\end{array}$ & $\begin{array}{l}\text { Pop/ } \\
11-20\end{array}$ & $\begin{array}{l}\text { Pop/ } \\
21-50\end{array}$ & $\mathrm{Fau} / 0$ & $\mathrm{Fau} / \mathrm{s}$ & $\mathrm{Fau} / \mathrm{d}$ & $\mathrm{Fau} / \mathrm{ds}$ & For & Sav & $\mathrm{Cab}$ & Mai & $\begin{array}{l}\mathrm{Sol} / \\
\mathrm{MO}\end{array}$ & $\mathrm{Sol} / \mathrm{H}$ & Sol/C & $\mathrm{Sol} / \mathrm{P}$ \\
\hline Bakoumba (12) & 12 & 0 & 0 & 0 & 0 & 12 & 0 & 0 & 0 & 12 & 0 & 0 & 6 & 6 & 0 & 0 \\
\hline Fougamou (15) & 5 & 6 & 4 & 0 & 10 & 0 & 5 & 0 & 5 & 6 & 4 & 0 & 4 & 5 & 1 & 5 \\
\hline Franceville (15) & 3 & 7 & 5 & 0 & 4 & 0 & 4 & 7 & 0 & 6 & 9 & 0 & 3 & 0 & 1 & 11 \\
\hline Libreville (12) & 0 & 0 & 0 & 12 & 0 & 0 & 0 & 12 & 0 & 0 & 0 & 12 & 4 & 0 & 0 & 8 \\
\hline Makokou (15) & 0 & 15 & 0 & 0 & 0 & 15 & 0 & 0 & 15 & 0 & 0 & 0 & 15 & 0 & 0 & 0 \\
\hline Malouma (14) & 12 & 2 & 0 & 0 & 12 & 0 & 2 & 0 & 10 & 0 & 4 & 0 & 12 & 0 & 2 & 0 \\
\hline Mouila (16) & 9 & 7 & 0 & 0 & 9 & 0 & 7 & 0 & 0 & 9 & 7 & 0 & 0 & 9 & 0 & 7 \\
\hline Ntoum (15) & 0 & 15 & 0 & 0 & 0 & 15 & 0 & 0 & 0 & 9 & 6 & 0 & 0 & 0 & 5 & 10 \\
\hline
\end{tabular}

Tableau III. - Distribution des pièges entre les différentes modalités de paramètres (abréviations : voir tableau I).

$S$. niger niger est l'espèce la plus abondante à Franceville, Ntoum et Fougamou, mais elle est aussi abondante dans trois autres localités : Bakoumba, Mouila et Libreville. À Franceville, S. transvittatus est peu abondante. En revanche, cette espèce est bien représentée dans toutes les autres localités (en particulier à Makokou et Malouma). S. calcitrans présente des effectifs très inégaux dans les huit localités prospectées. En effet, cette espèce, qui représente entre 25 et 50 \% des captures à Franceville, Libreville et Ntoum, représente moins de $12,5 \%$ des captures dans quatre autres localités. S. inornatus est présent dans toutes les localités, mais les captures sont partout faibles (<12,5\%). S. omega présente un schéma similaire, mais n'a pas été capturé à Libreville. S. xanthomelas n'a été capturé, en faible abondance, qu'à Makokou et Ntoum.

Le tableau III indique pour chaque localité la distribution des pièges utilisés en fonction des modalités des facteurs environnementaux envisagés. Les paramètres utilisés pour décrire l'environnement des pièges expliquent respectivement $87 \%$ et $77,5 \%$ de la distribution des espèces le long des deux premiers axes de l'analyse canonique des correspondances (figure 4). Les distributions spatiales de $S$. niger niger et $S$. transvittatus (espèces assez abondantes et largement distribuées) ainsi que de $S$. inornatus et $S$. omega (espèces rares et largement distribuées) ne sont pas fortement caractérisées par l'analyse. On peut remarquer néanmoins que l'anthropisation est plus favorable à $S$. niger niger (au-dessus de l'axe horizontal) qu'à S. transvittatus (au-dessous de l'axe horizontal). En revanche, l'ACC montre clairement que : 1) la présence de pâturages est favorable à $S$. calcitrans, 2) $S$. $n$. bilineatus semble assez étroitement lié à la faune sauvage savanicole et 3) S. xanthomelas est inféodé aux forêts.

\section{DISCUSSION}

I es résultats obtenus dans cette étude constituent les premières données concernant les stomoxes au Gabon. Le piège Vavoua, qui a été utilisé au cours de ce travail, semble être sélectif et présente donc l'avantage de capturer essentiellement le groupe cible. En effet, dans notre étude, les stomoxes ont représenté plus de $73 \%$ de l'ensemble des captures. Bien que l'efficacité du piège Nzi pour la capture des Muscidae piqueurs soit importante (Mihok, 2002), les résultats obtenus dans notre étude confirment l'efficacité du piège Vavoua pour la capture des stomoxes (Mihok, 1995; Gilles et al., 2007)

Au total sept espèces ou sous-espèces ont été identifiées dans les captures, ce qui représente $50 \%$ des espèces connues en région afrotropicale (Zumpt, 1973). 


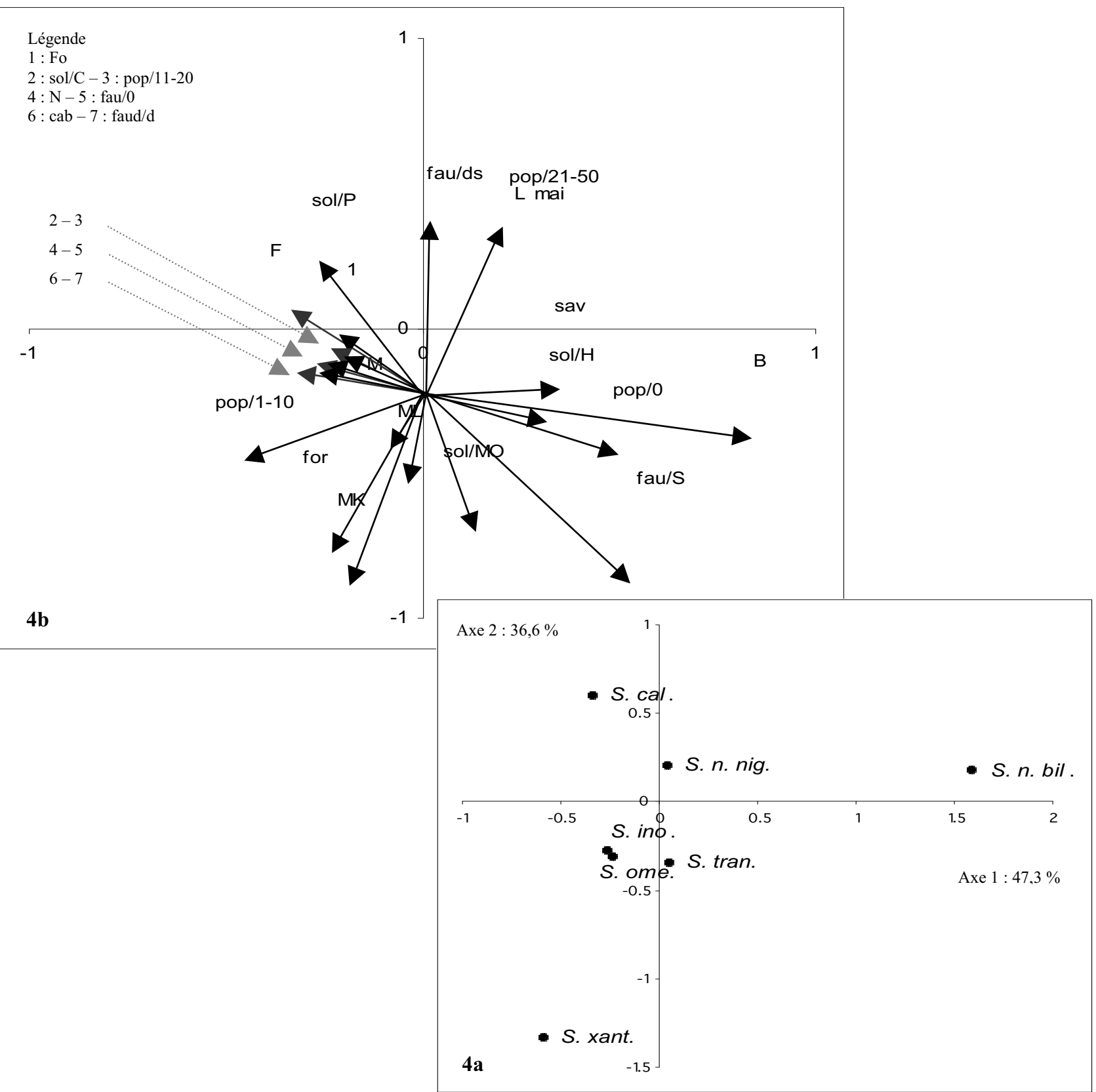

Fig. 4. - Diagramme d'ordination de l'ACC (plan des axes 1 et 2). La figure principale (4a) indique la distribution des espèces dans le plan (S. tran. : S. transvittatus, S. ino. : S. inornatus, S. xant. : S. xanthomelas, S. cal. : S. calcitrans, S. n. nig. : S. niger niger, S. ome. : S. omega, $S$. n. bil. : S. niger bilineatus). La figure complémentaire (4b) indique les corrélations (vecteurs) des modalités des différents paramètres environnementaux avec les deux premiers axes (abréviations conformes au tableau I et à la figure 2).

Mihok et al. (1996b) avaient capturé neuf espèces ou sous-espèces au Kenya (S. varipes Bezzi, S. n. bilineatus Grünberg, S. inornatus Grünberg, S. taeniatus Bigot, S. niger niger Macquart, S. transvittatus Villeneuve, S. taeniatus f. brunnipes Grünberg, S. pallidus Roubaud et $S$. calcitrans L.). Cela traduit-il une richesse spécifique plus faible au Gabon? On ne peut pas conclure pour le moment, car un seul type de piège a été utilisé dans notre étude et seuls 8 sites ont été inventoriés pendant une période relativement courte. D'autres captures sont nécessaires avec plusieurs types de pièges et d'attractifs, dans un plus grand nombre de sites et sur une période plus longue, pour avoir une image plus précise de la richesse spécifique des stomoxes au Gabon.

L'analyse des captures a montré que certaines espèces ( $S$. n. niger et $S$. transvittatus) étaient plus abondantes que les autres et que certains sites présentaient des DAP plus importantes (Franceville, Bakoumba, Makokou, et Mouila). L'espèce $S$. $n$. niger a été relevée dominante à Franceville, Mouila, Bakoumba, Libreville, 
Ntoum et Fougamou. S. calcitrans est fortement présente dans quatre localités (Franceville, Libreville, Ntoum, Fougamou). Ces localités sont caractérisées par une forte action anthropique liée à la présence de cases d'habitation humaine, d'animaux domestiques et de pâturages. Ces observations sont confirmées par l'analyse des facteurs environnementaux dans l'ACC. L'espèce $S$. niger niger serait l'espèce la plus répandue dans les zones de savanes et les forêts tropicales humides africaines (Zumpt, 1973). Elle semble abondante dans le centre du Kenya (Mihok, 1996b) et autour des campements d'éleveurs du Sud Soudan (Zumpt, 1973). Concernant l'espèce $S$. calcitrans, on sait qu'il s'agit d'une espèce cosmopolite souvent associée aux activités humaines liées à l'élevage (Zumpt, 1973 ; Gilles, 2005). S. xanthomelas est présente, en faible abondance, dans la zone forestière de Makokou et de Ntoum. Zumpt (1973) indique l'extrême rareté de cette espèce dans les collections et l'absence totale d'information sur sa biologie. Les résultats obtenus au cours d'une autre étude au Gabon (Mavoungou et al., 2007) semblent montrer que $S$. xanthomelas vivrait dans la canopée de la forêt équatoriale. Des résultats préliminaires concernant l'origine des repas de sang (50\% sur le singe cercopithèque "moustac" Cercopithecus cephus (Linnaeus, 1758)) sont en faveur d'une niche écologique dans la canopée pour cette espèce (Mavoungou et al., soumis). Les espèces $S$. tranvittatus, $S$. omega et $S$. inornatus semblent plus inféodées au milieu forestier où la densité humaine est faible et le sol recouvert de matière organique. Ces résultats rejoignent les observations de Mihok et al. (1996b) au Kenya.

Le microclimat pourrait aussi jouer un rôle dans la répartition et l'abondance des différentes espèces de stomoxes, mais les données précises manquent sur ce point. En effet Gilles et al. (2005a, 2005b) ont montré que la température avait un effet sur le taux de développement de deux espèces de stomoxes ( $S$. calcitrans, et $S$. n. niger). En étudiant la répartition des populations de l'araignée sociale Agelena consociata Denis à Makokou, Darchen (1978) avait enregistré des données thermométriques dans trois milieux : forêt, clairière et piste (milieu ouvert). Il avait établi que la température moyenne était variable dans les trois milieux. En forêt, elle oscille entre 20 et $24^{\circ} \mathrm{C}$ tandis qu'en milieu ouvert elle se situerait entre 23 et $28^{\circ} \mathrm{C}$.

Au total, cette première étude concernant les stomoxes au Gabon a permis de mettre en évidence sept espèces ou sous-espèces dans huit localités différentes. L'analyse des facteurs environnementaux caractérisant les lieux de capture a permis de mettre en évidence des relations entre certaines de ces espèces et des paysages particuliers. Une étude plus approfondie de la distribution spatio-temporelle de ces espèces a été entreprise sur un transect forêt-village à proximité de la station de Makokou (Mavoungou, 2007).

\section{REMERCIEMENTS}

C e travail a été réalisé grâce à un financement de l'Union européenne via le Projet Fonds Européen de Développement 8 ACP GA 009 (PSVAP - Composante II Makokou, CNPN-MESRIT-U.E./IRETCIFOR). Nous tenons à remercier Philippe Hecketsweiller, Paul Posso, Robert Nasi et Charles Doumenge pour leurs conseils et leur aide. Merci à Nicolas Yao Emba, Aimé Roger Nzigou pour leur aide technique.

\section{RÉEÉRENCES}

Basset Y., Mavoungou J.F., Mikissa J. B., Missa O., Miller S.E., KITChing R.L. \& Alonso A. Discriminatory power of different arthropod data sets for the biological monitoring of anthropogenic disturbance in tropical forests. Biodiversity and Conservation, 2004, 13, 709-732.

Charlwood J.D. \& Sama S. The age structure, biting cycle and dispersal of Stomoxys niger Macquart (Diptera: Muscidae) from Ifakara, Tanzania. African Entomology, 1996, 4 (2), $274-277$

DARChEN R. Les populations d'Agelena consociate Denis, araignée sociale, dans la forêt primaire gabonaise. Leur répartition et leur densité. Annales de sciences naturelles, Zoologie, Paris 1978, 14 (2), 19-26.

EDICEF. Géographie et cartographie du Gabon. Edicef, Paris, 1983.

FolL L.D. \& Gorham J. Mechanical transmission of disease agents by arthropods, in: Medical Entomology. Eldridge B.F. \& Edman J.D. (eds), Dordrecht, The Netherlands, Kluwer Academic Publishers, 2000, 461-514

Garros C., Gilles J. \& Duvallet G. Un nouveau caractère morphologique pour distinguer Stomoxys calcitrans et $S$. niger (Diptera : Muscidae). Comparaison de populations de l'île de la Réunion. Parasite, 2004, 11, 329-332.

GILlEs J. Dynamique et génétique des populations d'insectes vecteurs. Les Stomoxes, Stomoxys calcitrans et Stomoxys niger niger dans les élevages bovins réunionnais. [Thèse de Doctorat], Université de la Réunion, 2005.

Gilles J., David J.F. \& Duvallet G. Temperature effects on the development and survival of two stable flies from Stomoxys calcitrans and Stomoxys niger niger (Diptera: Muscidae) in La Réunion island. Journal of Medical Entomology, 2005a, 42 (3), 260-265.

Gilles J., David J.F. \& Duvallet G. Effects of temperature on the rate of increase of two stable flies from La Réunion island, Stomoxys calcitrans and Stomoxys niger niger (Diptera: Muscidae). Journal of Medical Entomology, 2005b, 42 (6), 959-965.

Gilles J., David J.F., Duvallet G., De la Rocque S. \& Tillard E. Efficiency of traps for Stomoxys calcitrans and Stomoxys niger on La Reunion Island. Medical and.Veterinary. Entomology, 2007, 21, 65-69.

Hafez M. \& GAMAL-EdDin F.M. Ecological studies on Stomoxys calcitrans L. and sitiens Rond. in Egypt, with suggestions 
on their control. Bulletin de la Société d'Entomologie d'Egypte, 1959a, 43, 245-254.

Hafez M. \& Gamal-Eddin F. M. On the feeding habits of Stomoxys calcitrans $\mathrm{L}$. and sitiens Rond. with special reference to their biting cycle in nature. Bulletin de la Société d'Entomologie d'Egypte, 1959b, 43, 291-301.

Hafez M. \& Gamal-Eddin F.M. The behaviour of the stable fly larva, Stomoxys calcitrans L., towards some environmental factors. Bulletin de la Société d'Entomologie d'Egypte, 1961, 45, 341-367.

HARLEY J.M.B. Seasonal abundance and diurnal variations in activity of some Stomoxys and Tabanidae in Uganda. Bulletin of entomological Research, 1965, 55, 319-332.

Kangwagye T.N. Diurnal and noctural biting activity of flies (Diptera) in western Uganda. Bulletin of entomological Research, 1973, 63, 17-29.

LAVEssière C. \& GréBAut P. The trapping of tsetse flies (Diptera: Glossinidae). Improvement of a model: the Vavoua trap. Tropical Medicine and Parasitology, 1990, 41 (2), 185192.

LECLERCQ M. Les mouches nuisibles aux animaux domestiques. Presses agronomiques de Gembloux, 1971.

Mavoungou J.F. Écologie et rôle vecteur des stomoxes (Diptera : Muscidae) au Gabon. [Thèse de Doctorat], Université Paul-Valéry Montpellier 3, 2007.

Mavoungou J.F., Gilles F. \& Duvallet G. Stomoxys xanthomelas Roubaud, 1937 (Diptera : Muscidae) : une espèce de la canopée en Afrique équatoriale? Bulletin de la Société Entomologique de France, 2007, 4, 481-483.

Mavoungou J.F, Simo G., Gilles G. \& Duvallet G. Écologie des stomoxes (Diptera : Muscidae) au Gabon. II - Origine des repas de sang et conséquences épidémiologiques. Parasite (soumis).

Minok S., Kang'ethe E.K. \& Kamau G.K. Trials of traps and attractants for Stomoxys (Diptera: Muscidae). Journal of Medical Entomology, 1995b, 32, 283-289.

Minok S., Maramba O., Munyoki E. \& Kagoiya J. Mechanical transmission of Trypanosoma spp. by African Stomoxyinae. Tropical Medecine and Parasitilogy, 1995a, 46, 103-105.

Minok S. \& Clausen P.H. Feeding habits of Stomoxys spp. stable flies in a Kenyan forest. Medical and Veterinary Entomology, 1996a, 10, 392-394.

Minok S., Opiyo M., Eli M. \& Khalfan S. Phenology of Stomoxinae in a Kenya forest. Medical and Veterinary Entomology, 1996b, 10, 305-316.

Mıнок S. The development of a multipurpose trap (the Nzi) for tsetse and other biting flies. Bulletin of Entomological Research, 2002, 92, 385-403.

Richard A. \& LÉOnARD G. Le Gabon. EDICEF/EDIG, Vanves/ Libreville, 1993, 287 p.

TER BRAAK C.J.F. Canonical correspondence analysis: a new eigenvector technique for multivariate direct gradient analysis. Ecology, 1986, 67 (5), 1167-1179.

TER BraAK C.J.F. \& SMILAUER P. CANOCO Reference manual and CanoDraw for Windows User's guide: Software for Canonical Community Ordination (version 4.5). Microcomputer Power, Ithaca, USA, 2002.
Zumpt F. The Stomoxyine Biting Flies of the World. Diptera: Muscidae. Taxonomy, biology, economic importance and control measures. Stuttgart: Gustav Fisher Verlag, 1973.

Reçu le 27 septembre 2007 Accepté le 29 octobre 2007 